\title{
Congratulatory message
}

\section{Sir David Cox ${ }^{1}$}

Published online: 31 August 2018

(c) Springer Nature Singapore Pte Ltd. 2018

The systematic use of geometric ideas in connection with the distributional problems of statistical theory goes back at least to R. A. Fisher, perhaps most strikingly in his concise and powerful discussion of the formal distribution theory of linear discriminant analysis. Many years later Bradley Efron made an important contribution discussing the role of curvature of models. Very striking and influential, however, were the powerful and highly original papers and lectures of Professor Shun-ichi Amari in the University of Tokyo. Statistical theory at the moment is faced with major challenges arising from the scale of many modern investigations and developments must hinge in part in new developments in asymptotic theory, surely having a new and challenging geometric basis. This makes the launch of a new journal highly appropriate. It will be influential and successful.

Sir David Cox

david.cox@nuffield.ox.ac.uk

1 Nuffield College, University of Oxford, New Road, Oxford OX1 1NF, UK 